\title{
Simulation of Cold Flow in a Truncated Ideal Nozzle with Film Cooling
}

\author{
Kalen Braman* \\ Tuskegee University, Jacobs ESSSA Group, NASA Marshall Space Flight Center, Huntsville, AL 35812, \\ USA \\ Joseph Ruf** \\ NASA Marshall Space Flight Center, Huntsville, AL 35812, USA
}

\begin{abstract}
Flow transients during rocket start-up and shut-down can lead to significant side loads on rocket nozzles. The capability to estimate these side loads computationally can streamline the nozzle design process. Towards this goal, the flow in a truncated ideal contour (TIC) nozzle has been simulated for a range of nozzle pressure ratios (NPRs) aimed to match a series of cold flow experiments performed at the NASA MSFC Nozzle Test Facility. These simulations were performed with varying turbulence model choices and with four different versions of the TIC nozzle model geometry, each of which was created with a different simplification to the test article geometry.
\end{abstract}

\section{Introduction}

The design of new rocket nozzles or the use of existing designs in new configurations requires the estimation of nozzle transient effects such as occur during start-up and shut-down. These transients can cause significant side loads on the nozzle. The relative magnitude of these side loads for different types of nozzle contours can be estimated with subscale cold flow experiments. However, determining the absolute magnitudes of nozzle side loads requires full scale hot fire tests. These hot fire tests can be costly with regards to both money and time, especially if design problems are not discovered until the full scale tests. Therefore, a computational tool which can predict these transient effects can greatly streamline the design and test cycle.

An accurate prediction of the nozzle side loads requires an accurate prediction of the nozzle separation location. Furthermore, the separation location can be affected by nozzle modifications, like the film cooling systems applied in radiatively cooled nozzles [1]. Thus a computational tool capable of accurately predicting nozzle separation must be able to account for these nozzle modifications.

This paper addresses the computational modeling requirements necessary to predict the effects of nozzle film cooling on the surface pressures and separation location in a nozzle. Focus of this work includes the simplifications applied to the computational geometry, as well as the turbulence modeling choices made, and how these affect the surface pressure and separation location accuracy. To facilitate the discussion of solution accuracy, a nozzle with corresponding experimental results was selected. Specifically, the nozzle geometry aims to match that used during a series of cold flow nozzle experiments performed at the NASA MSFC Nozzle Test Facility (NTF) [2,3]. In this set of experiments, the nozzle pressure ratio (NPR) was stepped through a range of stationary values by decreasing the facility back pressure. The aim is to show

\footnotetext{
* Combustion Instability and Fluids Analyst $* *$
} 
what level of geometry simplifications and which turbulence modeling choices provide reasonable accuracy in determining surface pressures and separation location.

\section{Methodology}

\subsection{Model Geometry}

The simulation models were derived from a truncated ideal contour (TIC) nozzle geometry [2,3]. The nozzle geometry includes an annular slot in the diverging section, the 'nozzlette', which provides an auxiliary supersonic flow injection for film cooling. Figure 1 shows a schematic of the nozzle and nozzlette. A more detailed view of the nozzlette is shown in Fig. 2.

One axisymmetric and three different three-dimensional (3D) simulation models were created to approximate the TIC nozzle geometry. The four different computational models aimed to determine the sensitivity of the simulation results to the approximations made in defining the computational model geometries and computational grids. Additional computational grids were created to test grid density effects but were not included in the tally of four.

The axisymmetric grid uses an equivalent slot area to mimic the effect of the flow blockage caused by the teeth, since the orifice teeth cannot be created in an axisymmetric grid. The first of the three 3D computational grids, labeled 3D_twoD_tooth, was created by rotationally extruding the axisymmetric grid. The two periodic planes of 3D_twoD_tooth match exactly the axisymmetric grid. Like the axisymmetric grid, this grid contains none of the orifice teeth, but instead contains an obstruction which extends rotationally around the orifice. The second 3D grid, labeled 3D_sqr_tooth, again was created by extrusion, except for the nozzlette tooth region. For this grid, the rounded tooth design of the TIC nozzlette geometry was replaced with a squared tooth design. The squared tooth design aimed to provide 3D blockage to the nozzlette flow equivalent to that anticipated with a rounded tooth while maintaining the ability to use a structured grid in the nozzlette orifice region. The final 3D grid, labeled 3D_rnd_tooth, includes a tooth and channel which match the design of the TIC nozzlette. The bulk of the computational domain of these last two grids match exactly grid 3D_blockage. The difference shows only in the nozzlette region. All three 3D grids span a single tooth/channel section azimuthally.

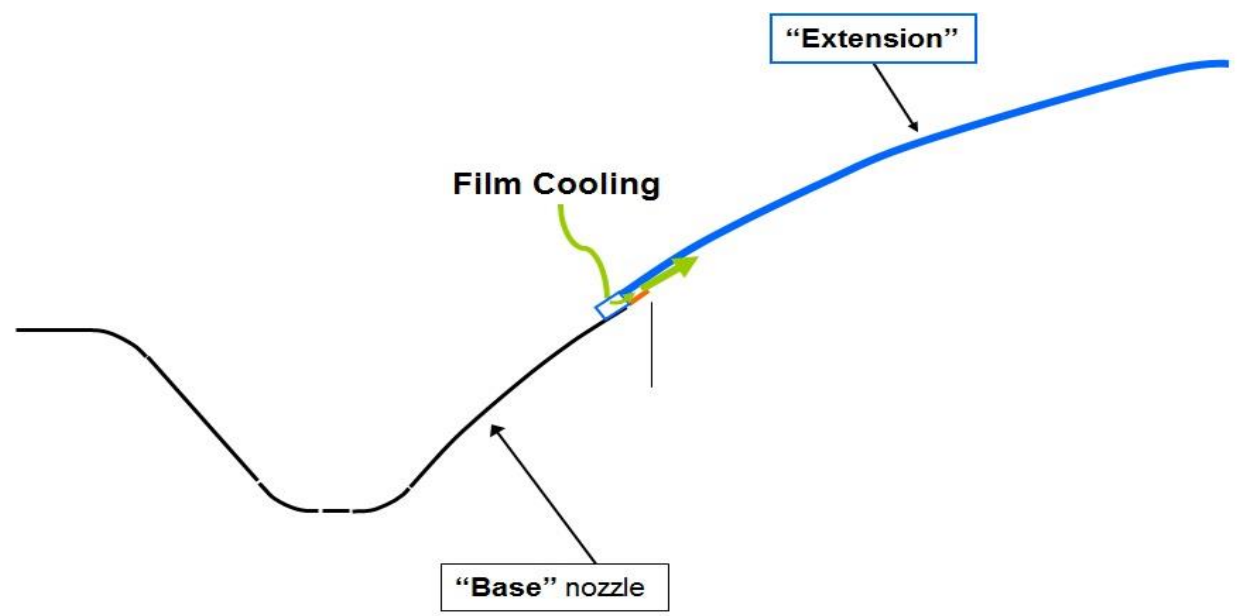

Figure 1: The TIC nozzle approximate contour with the film cooling nozzlette 


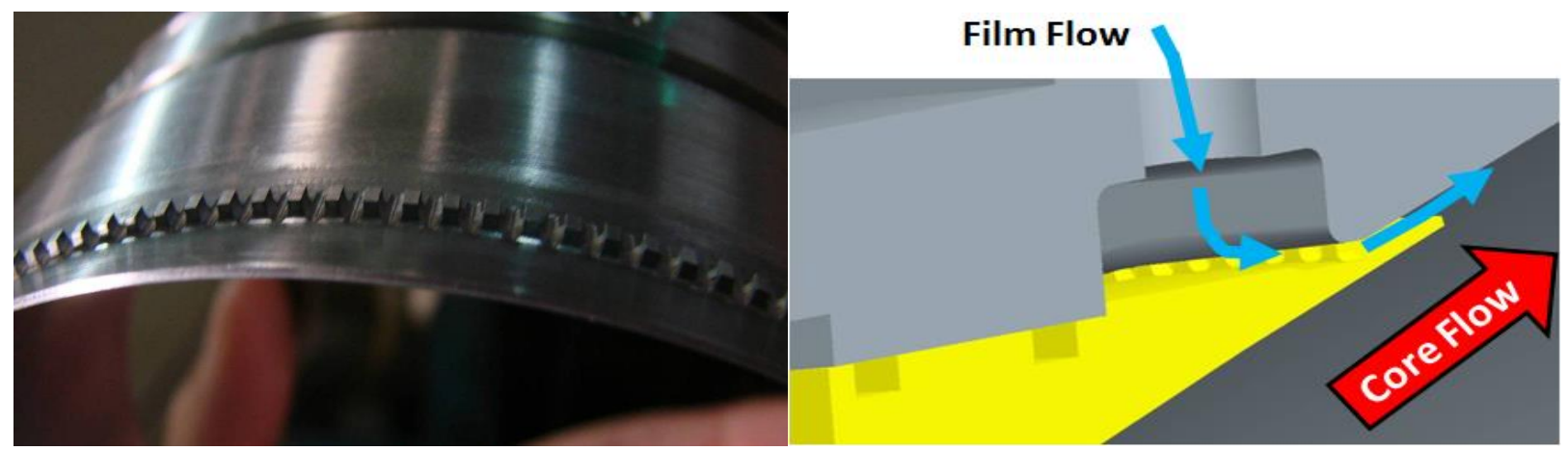

Figure 2: The nozzlette geometry with an upstream view of the actual hardware (left) and an isometric view of the manifold and nozzlette orifice (right)

\subsection{Simulation Methodology}

The simulations were performed to steady state using Loci/CHEM. Loci/CHEM [4 -6] is a density-based finite-volume CFD program built upon the Loci [7,8] framework. To reach steady state in fewer iterations, a two stage process was used involving changes to a limited number of boundary conditions and Loci/CHEM settings. For each different axisymmetric grid configuration, the corresponding simulations were stepped forward through NPR using the result of the previous NPR's simulation as the initial condition. For each 3D grid configuration, the simulation started using the corresponding axisymmetric steady solution as the initial condition. The turbulence models included in this investigation are Menter's baseline (BSL) $[9,10]$ and Menter's Shear Stress Transport (SST) $[9,10]$ models, and the compressibility corrections are those by Sarkar [11] and Wilcox [12].

\section{Preliminary Results}

The simulations presented here aim to approximate a set of experiments performed at the MSFC NTF $[2,3]$. In the experiments, heated dry air was delivered to the test article. Experiments were conducted over a range of NPRs by varying the pressure in the test cell through the use of an ejector system. Two auxiliary nozzlette flow rates are documented here, no film flow and supersonic film flow.

The experimental measurements included a dense array of static pressures along the nozzle surface $[2,3]$. These measurements of pressure allow the determination of the nozzle wall separation location. Thus, the primary quantities of interest for the simulations are the nozzle surface pressures and the locations of separation. For the remainder, the surface pressure distributions will be reported normalized by chamber pressure and plotted versus axial distance normalized by the nozzle throat radius $r^{*}$.

Results for the axisymmetric grid were computed for a set of turbulence modeling choices which are listed in Table 1. Each of these selections was performed for both auxiliary flow cases. Figures 3 and 4 display the pressure profiles for no auxiliary nozzlette flow and supersonic nozzlette flow, respectively. For no nozzlette flow, case A1 which uses Menter's BSL turbulence model and the Sarkar compressibility correction, generally matches the experimental results the closest. The other three model combinations show early separation for every NPR, whereas A1 matches the pressures very closely for NPR 110, shows late separation for lower NPRs, and early separation for higher NPRs. For the cases with supersonic 
nozzlette flow, A2 tends to match experiments the closest, with late separation at NPR below 50 and early separation with NPR above 50. A3 and A4 show early separation for every NPR except 15, and A1 shows late separation for NPRs below 145.

Table 1: Turbulence model selections for the axisymmetric simulations

\begin{tabular}{|c|c|c|}
\hline Case & Turbulence model & Compressibility correction \\
\hline A1 & BSL & Sarkar* \\
\hline A2 & BSL & Wilcox \\
\hline A3 & SST & Sarkar* \\
\hline A4 & SST & Wilcox \\
\hline
\end{tabular}

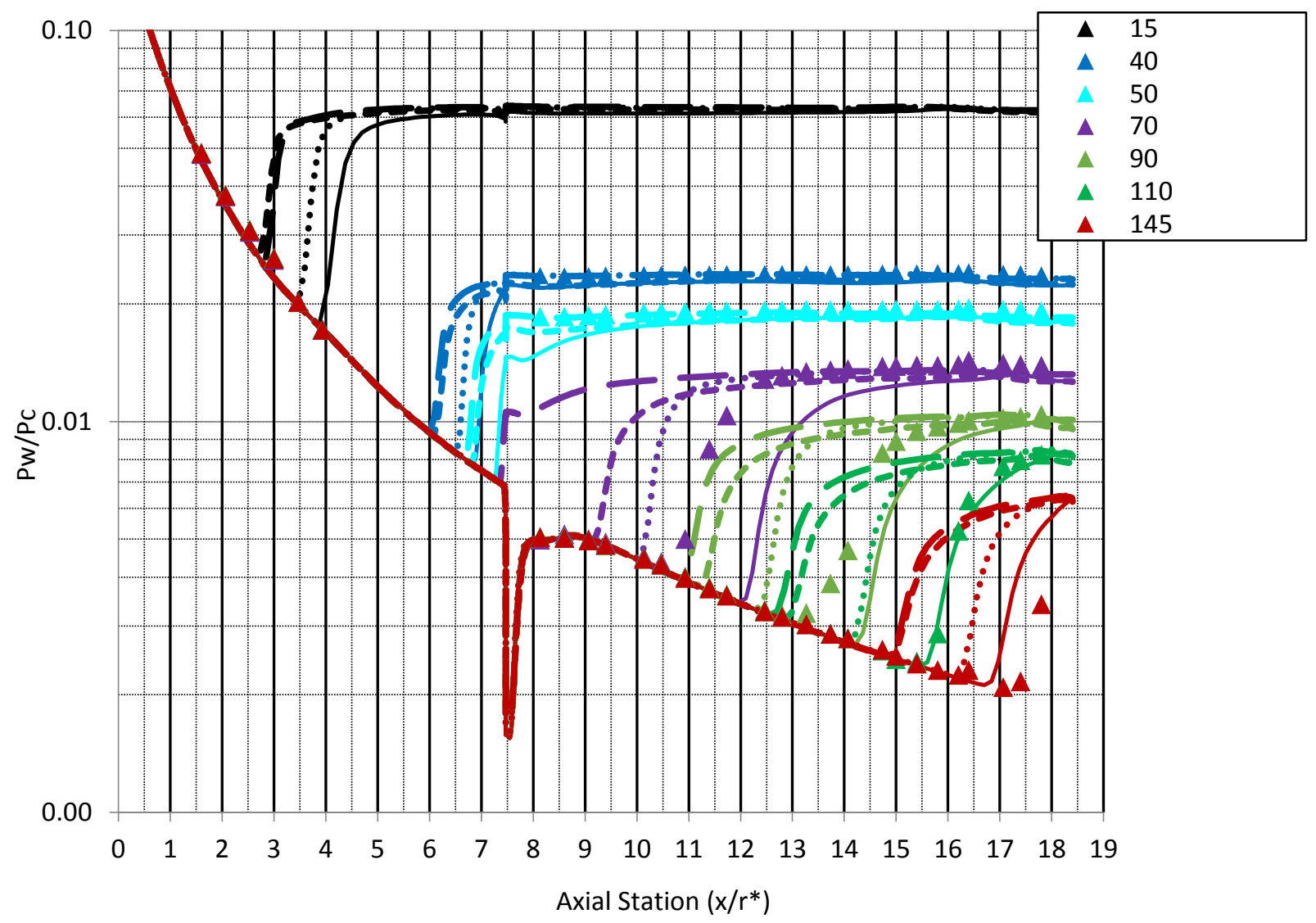

Figure 3: Non-dimensional pressure profiles for the axisymmetric grid with no auxiliary nozzlette flow with case A1 solid lines, case A2 dotted lines, case A3 dashed lines, and case A4 long dashed lines, plotted against the experimental results in symbols 


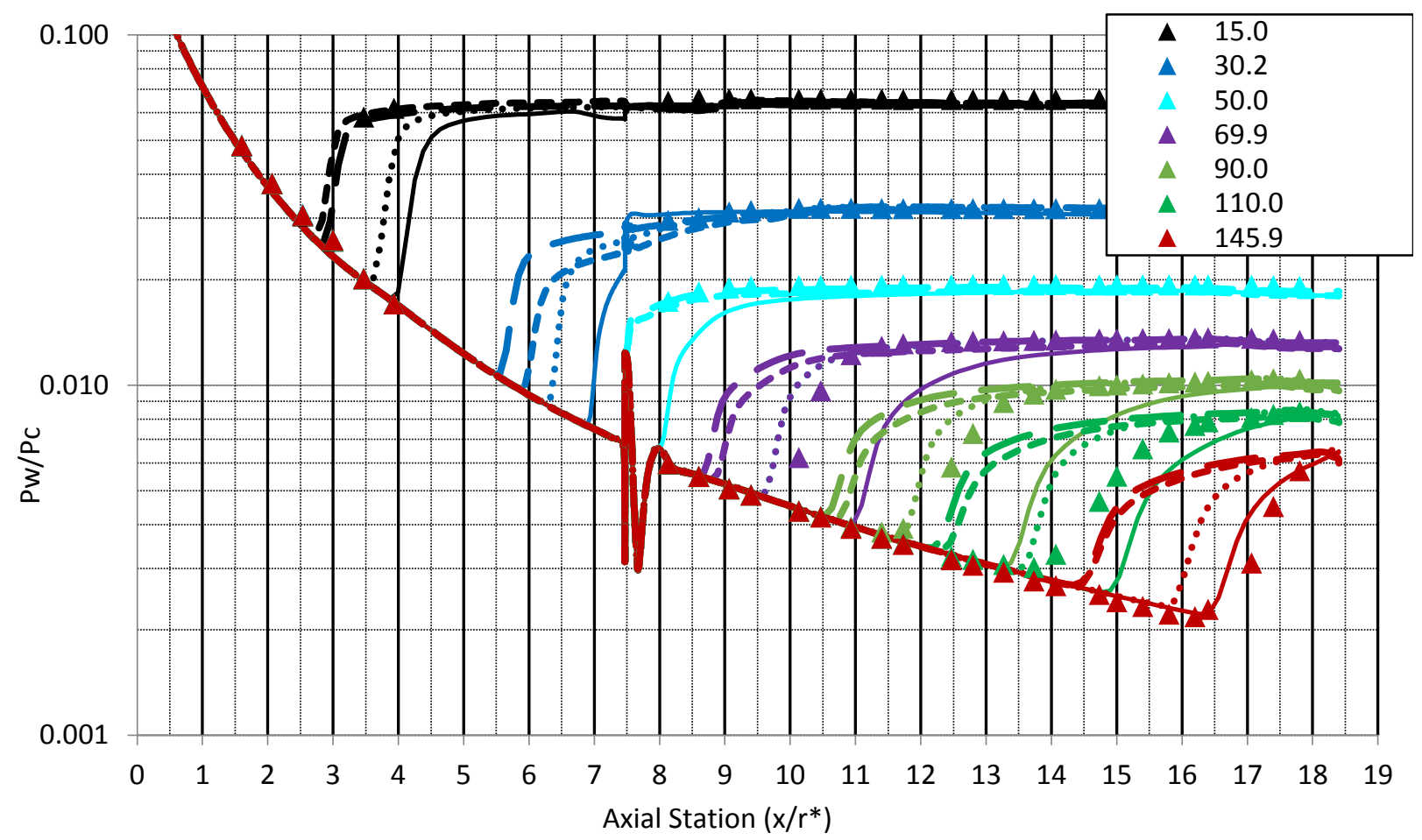

Figure 4: Non-dimensional pressure profiles for the axisymmetric grid with supersonic auxiliary nozzlette flow with case A1 solid lines, case A2 dotted lines, case A3 dashed lines, and case A4 long dashed lines, plotted against the experimental results in symbols

Preliminary results for the 3D grids were all computed using the same turbulence model selection, Menter's BSL model with the Sarkar* compressibility correction, with supersonic nozzlette flow. These results are displayed against the corresponding axisymmetric results and the experimental results in Figure 5. For NPRs 15 and 40, when separation occurs upstream of the nozzlette exit, all computational models produce the same separation location. For NPRs 50 and 60, the different models produce different separation locations. The axisymmetric model and 3D_blockage match very closely, which is expected given that the 3D model is the axisymmetric model rotationally extruded. The models which involve 3D geometric features in the film coolant path tend to show separation farther downstream. This effect is caused by the penetration of the nozzlette film cooling. For the cases involving 3D features, the film cooling flow remains focused in a narrower 'jet', allowing it to penetrate further into the main nozzle. The greater penetration of the nozzlette's momentum, as compared to that for the models with 2D nozzlette features, delays the point of separation farther down the nozzle surface. An exception to this trend occurred with 3D_sqr_tooth at NPR 50, which showed separation still at the nozzlette exit. When compared to the experimental results, the axisymmetric and 3D blockage models match better than the grids which incorporate 3D features. This does not imply that the blockage models are a better representation of reality. This only implies that the set of modeling choices and geometry simplifications made in defining the problem lead to a better match with experiment for the blockage models. 

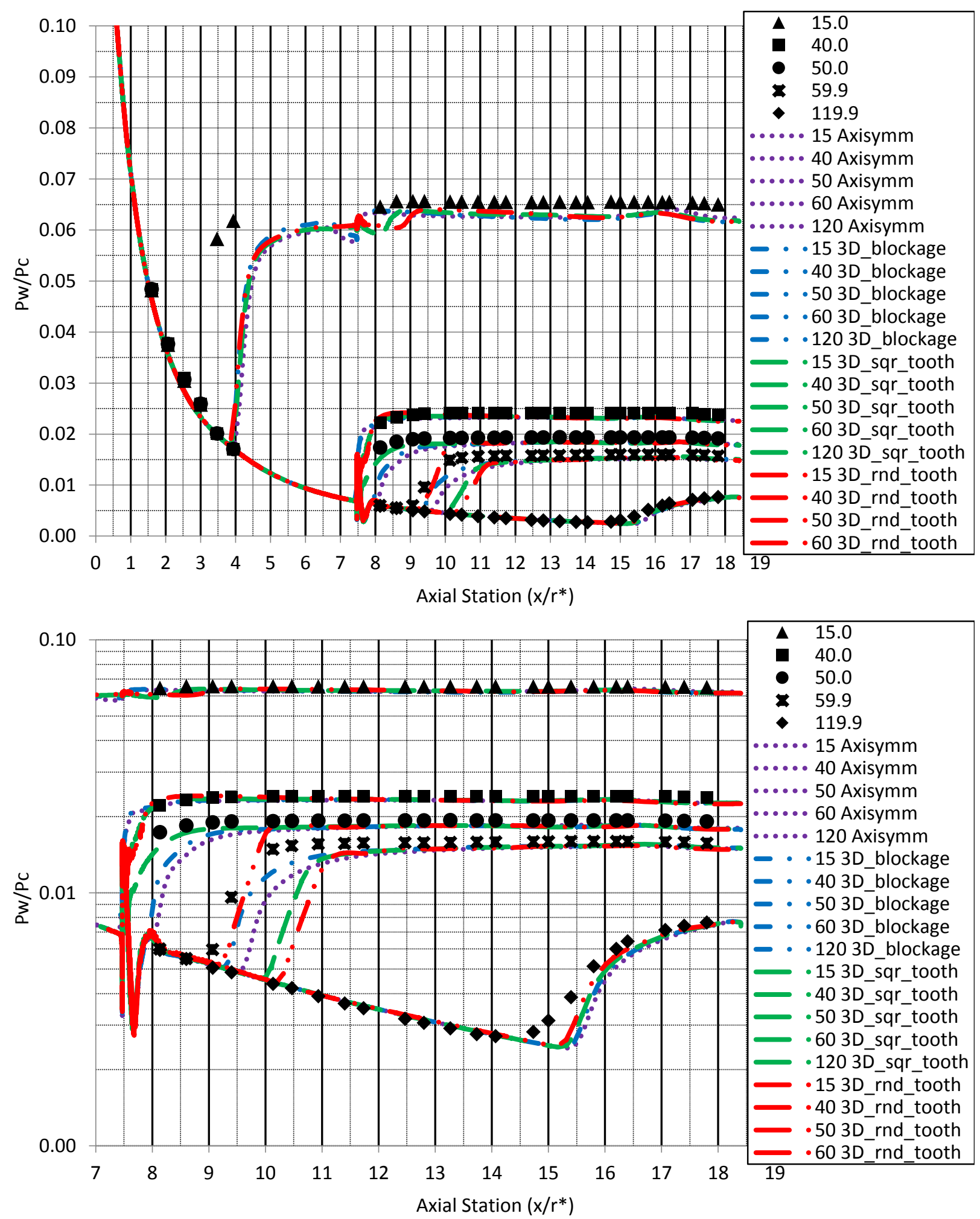

Figure 5: Non-dimensional pressure profiles at various NPR for all grids using Menter's BSL model and Sarkar* compressibility correction plotted against the experimental results 


\section{Preliminary Conclusions}

A series of axisymmetric and 3D grid topographies were developed to approximate the TIC nozzle film coolant injection geometry. These various models were created to determine the sensitivity of the results to the simplifications applied to the geometry for the film injection. The axisymmetric equivalent area slot geometry matches closely its 3D rotationally extruded counterpart (3D_twoD_tooth), thus little appears to be gained by treating the equivalent area slot case with a 3D grid. The simulations involving the two grids with 3D features, the squared-off tooth representation (3D_sqr_tooth) and the rounded tooth representation (3D_rnd_tooth), generally exhibit a longer delay to separation for the higher NPR cases than the equivalent slot area cases.

Generally, while the exact positions of the flow separation cannot be predicted by these simulations, the overall trend of the surface pressures and separation locations can be predicted fairly well. The results typically show one NPR at which the simulation's separation location matches the experiment, while lower NPRs show late separation (off by less than one nozzle throat radius, $\mathrm{r}^{*}$ ) and higher NPRs show early separation (off by less than $0.5 \mathrm{r}^{*}$ ).

\section{References:}

1. Martelli, E., F. Nasuti, and M. Onofri, "Film Cooling Effect on Dual-Bell Nozzle Flow Transition," AIAA Paper 2009-4953, Aug. 2009.

2. J. Ruf, D. McDaniels, and A. Brown, "Cold flow test results for nozzle side loads for J-2X and SSME test articles," JANNAF 2010.

3. J. Ruf, D. McDaniels, and A. Brown, "Cold flow test results for the effect of supersonic film cooling on a truncated ideal contour's nozzle side loads," JANNAF 2010.

4. E. Luke, X-L. Tong, J. Wu, L. Tang, and P. Cinnella, "A Step Towards `Shape Shifting'Algorithms: Reacting Flow Simulations Using Generalized Grids," 39th AIAA Aerospace Sciences Meeting and Exhibit, January 8-11, 2001, Reno, NV, AIAA Paper 2001-0897.

5. Q. Liu, E. Luke, and P. Cinnella, "Coupling Heat Transfer and Fluid Flow Solvers for MultiDisciplinary Simulations," AIAA Journal of Thermophysics and Heat Transfer, Vol. 19, No. 4, Oct.Dec. 2005, pp 417-427.

6. J. Wu, L. Tang, E. Luke, "A Low Mach Number Preconditioning Scheme of the Reactive Roe Flux," 41st AIAA Aerospace Sciences Meeting and Exhibit, January 6-9th, 2003, Reno, NV, AIAA Paper 2003-0307.

7. E. Luke, "A Rule-Based Specification System for Computational Fluid Dynamics," Ph.D. Dissertation, Mississippi State University, December, 1999.

8. E. Luke and T. George, "Loci: A Rule-Based Framework for Parallel Multidisciplinary Simulation Synthesis," Journal of Functional Programming, Special Issue on Functional Approaches to HighPerformance Parallel Programming, Volume 15, Issue 03, 2005, pp. 477-502, Cambridge University Press.

9. Menter, F.R., "Zonal Two Equation k-w Turbulence Models for Aerodynamic Flows,” AIAA Paper 93-2906, July 1993. 
10. Menter, F.R., "Two-Equation Eddy-Viscosity Turbulence Models for Engineering Applications," AIAA Journal, Vol. 32, No. 8, Aug. 1994, pp. 1598-1605.

11. Sarkar, S. and Lakshmanan, B., "Application of a Reynolds Stress Turbulence Model to the Compressible Shear Layer," AIAA Journal, Vol. 29, No. 5, May 1991, pp. 743-749.

12. Wilcox, D.C., Turbulence Modeling for CFD, DCW Industries, 1998. 